\title{
Chapter 6: A Theory of Granular Partitions
}

Thomas Bittner and Barry Smith

\section{Introduction}

Imagine that you are standing on a bridge above a highway checking off the makes and models of the cars that are passing underneath; or a laboratory technician sorting samples of bacteria into species and subspecies; or you are making a list of the fossils in your museum. In each of these cases, you are employing a certain grid of labeled units, and you are recognizing certain objects as being located in those units. Such a grid of labeled units is an example of what we shall call a granular partition. We shall argue that granular partitions are involved in all naming, listing, sorting, counting, cataloguing, and mapping activities, activities that are performed by human beings in their traffic with the world. Partitions are the cognitive devices designed and built by human beings to fulfill these various listing, mapping, and classifying purposes.

\section{Types of Granular Partitions}

Some types of granular partition grids are flat and amount to nothing more than mere lists. Others are hierarchical, consisting of units and subunits, the latter being nested within the former. Some grids are built in order to reflect independently existing divisions on the side of objects in the world (such as the subdivision of hadrons into baryons and mesons). Others - for example, the partitions created by classifying organisms into phyla or kingdoms, or by electoral redistricting - are themselves such as to create the corresponding divisions on the side of their objects, and sometimes they create those very objects themselves. Quite different sorts of partitions - having units of different resolutions and effecting unifyings, slicings and reapportionings of different types - can be applied to the same domain of objects simultaneously. Members of the animal kingdom can be divided according to what they eat, where they are indigenous, or even by the number of appendages an individual animal has. Maps, too, can impose subdivisions of different types upon the same domain of spatial reality, and the icons which they employ represent objects in granular fashion (which means that they do not represent the corresponding object parts). 
The theory of partitions is highly general, and this generality brings a correspondingly highly general reading of the term object with it. Here, we take an object to be any portion of reality like an individual, a part of an individual, a class of individuals (for example, a biological species), a spatial region, a political unit (county, polling district, nation), or even the universe as a whole. An object in the partition-theoretic sense is everything existent that can be recognized by some unit of a partition.

Objects can be either of the bona fide or of the fiat sort (Smith, 2001). Bona fide objects are objects which exist (and are demarcated from their surroundings) independently of human partitioning activity. Fiat objects are objects which exist only because of such partitioning activity. In some cases, partition units recognize fiat objects, such as your right arm or Poland, which exist independently of human cognition but which have boundaries that depend upon our human demarcations. In other cases, fiat objects are created through the very projection of partition units onto a corresponding portion of reality. Partitions which themselves create fiat objects include, for example, the partitions created by our ordinary classification of fruits and vegetables. Once fiat objects have been created in this way, subsequent partitions may simply recognize them (without any object-creating effect), as do those partitions which recognize bona fide objects.

In first approximation, granular partitions can be conceived as the mereological sums of their constituent units. This conception is roughly analogous to David Lewis's (1991) conception of classes as the mereological sums of their constituent singletons (thus the set $\{1,2,3\}$, for example, is conceived by Lewis as the mereological sum of the three sets $\{1\},\{2\}$, and $\{3\})$. The units within a granular partition, however, may manifest a range of properties which the singletons of set theory lack. This is so because, where each singleton is defined in the obvious way in terms of its single member, a unit of a granular partition is determined by its label as well; and this means independently of any object which might fall within it. The units of a partition are what they are independently of whether there are objects located within them. A map of Middle Earth is different from a map of the Kingdom of Zenda, even though, in both cases, there is nothing on the side of reality upon which these maps would be projected.

Just as when we point our telescope in a certain direction we may fail to find what we are looking for, so too, when we point our partition in a certain direction it may be that there are no objects located in its units. But 
this does not mean that the theory of partitions recognizes some counterpart of the set theorist's empty set (an entity that is contained as a subset within every set). For the empty set is empty by necessity; by contrast, a unit in a partition is empty per accidens at best.

The theory of Granular Partitions was originally developed in terms of first-order predicate logic, and a corresponding presentation of the theory in formal logical notation can be found in Bittner and Smith (2003). In what follows, however, symbolic notation is avoided as much as possible.

\section{Granular Partitions as Systems of Labeled Units}

\subsection{A Bipartite Theory}

In the present essay, we present the basic formal theory of granular partitions. Our formal theory has two orthogonal and independent parts, namely, (A) a theory of the relations between units, subunits, and the partitions in which they are contained, and (B) a theory of the relations between partitions and objects in reality. In a set-theoretic context, the counterpart of (A) would be the study of the relations among subsets of a single set, while the counterpart of (B), in the same context, would be the study of the relations between sets and their members. Partition theory departs from the extensionalism of set theory (that is, from the assumption that each set is defined exclusively by its members, so that two sets are identical if and only if they have the same members). A unit is defined by its position within a partition and by its relations to other units, and it is this which gives rise to the relations treated of by theory (A). What objects in reality are located in a unit - the matter of theory (B) - is a further question, which is answered in different ways from case to case. Briefly, we can think of units as being projected onto objects in something like the way in which flashlight beams are projected upon the objects which fall within their purview. For partition theory seeks to represent the ways in which cognitive agents categorize reality as it presents itself in all its unmathematical variety and scruffiness.

Consider the left part of Figure 1. Theory A governs the way we organize units into nesting structures and the way we label units. Theory B governs the way these unit-structures project onto reality, as indicated by the arrows connecting the left and the right parts of the figure. 
Figure 2: Relationships between Units and Objects

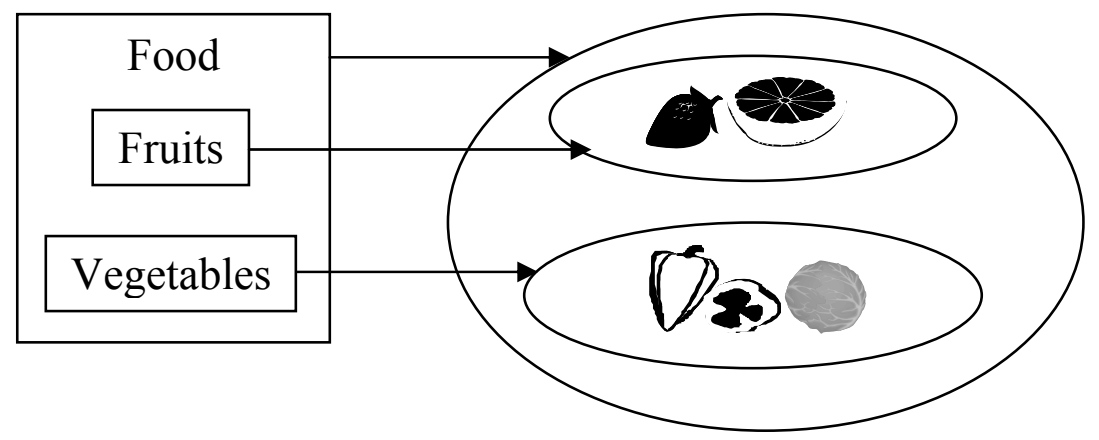

\subsection{The Subunit Relation}

Theory (A) is, effectively, a formation theory for partitions; it studies properties partitions have in virtue of the relations between, and the operations performed upon, the units out of which they are built independently of any linkage to reality beyond. Units in partitions may be nested one inside another in the way in which, for example, the species crow is nested inside the species bird in standard biological taxonomies. When one unit is nested inside another in this way, we say that the former is a subunit of the latter.

We use $\mathrm{z}, \mathrm{z}_{1}, \mathrm{z}_{2}, \ldots$ as variables ranging over units and $\mathrm{A}, \mathrm{A}_{1}, \mathrm{~A}_{2}, \ldots$ as variables ranging over partitions. We write ' $\mathrm{Z}_{1} \subseteq_{\mathrm{A}} \mathrm{Z}_{2}$ ' in order to express the fact that $z_{1}$ stands in a subunit relation to $z_{2}$ within the partition A. (Where confusion will not result, we will drop the explicit reference to the partition A and write simply ' $\subseteq$ '.) We can state the first of several master conditions on all partitions as follows:

MA1: The subunit relation $\subseteq$ is reflexive, anti-symmetric, and transitive.

This means that within every partition: each unit is a subunit of itself; if two units are subunits of each other, then they are identical; and if unit $z_{1}$ is a subunit of $z_{2}$ and $z_{2}$ a sub-unit of $z_{3}$, then $z_{1}$, in its turn, is a subunit of $z_{3}$.

\subsection{Existence of a Maximal Unit}

A maximal unit is the unit in a partition which encompasses all of the other subunits. (However, as we will see below, the maximal unit is not identical to the largest partition.) 
DMax: A unit $\mathrm{z}_{1}$ of partition A is a maximal unit if and only if every unit of $A$ is a subunit of $z_{1}$.

We now demand, as a further master condition, that

MA2: Every partition has a unique maximal unit in the sense of DMax.

The motivation for MA2 is very simple, and turns on the fact that a partition with two maximal units would either be in need of completion by some extra unit representing the result of combining these two maximal units together into some larger whole, or it would be two separate partitions, each of which would need to be treated in its own right.

MA2 implies that there are no partitions which are empty tout court in that they have no units at all. Although the maximal unit seems to be just as big as the partition itself, it is not identical to it; for the maximal unit is just that, a unit. Further, it is one that comprehends only a very large-grained perspective. By contrast the partition, itself, includes all the other subunits as its units as well.

\subsection{Finite Chain Condition}

The transitivity of the subunit relation generates a nestedness of units inside a partition in the form of chains of units, structured in such a way that maximal unit $z_{1}$ contains $z_{2}$, which contains $z_{3}$, all the way down to some $z_{n}$. We shall call the units at the lower ends of such chains minimal units (also called leaf nodes in the terminology of ontologies) and define:

DMin: $\mathrm{z}_{1}$ is a minimal unit of partition $\mathrm{A}$ if and only if every subunit $\mathrm{z}$ of partition $A$ which is a subunit of $z_{1}$ is also identical to $z_{1}$.

Another important aspect of a partition, then, is:

MA3: Each unit in a partition is connected to the maximal unit via a finite chain.

MA3 does not rule out the possibility that a given unit within a partition might have infinitely many immediate subunits, those which have no other units as intermediaries between themselves and their parent units. Thus MA3 enforces finite chains between the maximal partition and each of its units: every sequence of descending sub-units stops after a finite number of 
steps and there is only a finite number of such sequences; but this leaves open the issue of whether partitions themselves are finite.

If, in counting off the animals you saw in the rainforest, your checklist includes one unit labelled located in trees and another unit labelled has fur, we will rightly feel that there is something amiss with your partition. One problem is that you will almost certainly be guilty of double counting. Another problem is that there is no natural relationship between these two units, which seem rather to belong to distinct partitions. As a step towards rectifying such problems we shall insist that all partitions must satisfy the condition that every pair of distinct units within a partition are either related by the subunit relation or are disjoint. In other words:

MA4: If two units within a partition overlap, then one is a subunit of the other.

From MA3 and MA4 we can prove, by a simple reductio, that the chain connecting each unit of a partition to the maximal unit is unique.

\subsection{Partition-theoretic Sum and Product of Cells}

In this chapter, the background to all our remarks is mereology, which is the study of the relationships between wholes and their parts. We take the relation $\leq$ meaning 'part of' as primitive, and define the relation of overlap between two entities, simply, as the sharing of some common part. The part-of relation is like the subunit relation in being reflexive, antisymmetric, and transitive, but the two differ in the fact that the subunit relation is a very special case of the parthood relation.

The subunits of a unit are also parts of the unit just as, for David Lewis (1991), each singleton is a part of all the sets in which it is included. What happens when we take the mereological products and sums of units existing within a partition? In regard to the mereological product of two units, $\mathrm{z}_{1} * \mathrm{z}_{2}$, matters are rather simple. This product exists only when the units overlap mereologically, that is, only when they have at least one subunit in common. This means that the mereological product or intersection of two units, if it exists, is in every case just the smaller of the two units.

In regard to the mereological sum of units $\mathrm{z}_{1}+\mathrm{z}_{2}$, in contrast, a more difficult situation confronts us. Given any pair of units within a given partition, there is a corresponding mereological sum simply in virtue of the fact that the axioms of mereology allow unrestricted sum-formation. 
However, this mereological sum will be a unit within the partition in question only in special cases. This occurs, for example (and simplifying somewhat), when units labeled male rabbit and female rabbit within a partition have the unit labeled rabbit as their sum. By contrast, there is no unit labeled rabbits and jellyfish in our standard biological partition of the animal kingdom.

To make sense of these matters, we need to distinguish the mereological sum of two units from what we might call their partition-theoretic sum. Their mereological sum is the result of taking the two units together in our thoughts and treating the result as a whole, while their partition-theoretic sum consists of those mereological sums which we can recognize against the background of a given partition. The partition-theoretic sum of units $z_{1}$ and $z_{2}$ in a partition is the smallest subunit within the partition containing both $z_{1}$ and $z_{2}$ as subunits; that is, it is the least upper bound of $z_{1}$ and $z_{2}$ with respect to the subunit relation. (By MA2 and MA4, we know that this is always defined and that it is unique.) In general, this partition-theoretic sum is distinct from the mereological sum of the corresponding units. (The partition-theoretic sum of the units labelled rabbit and lion is the unit labelled mammal in our partition of the animal kingdom.) The best we can say, in general, is that the mereological sum of $z_{1}$ and $z_{2}\left(z_{1}+z_{2}\right)$ is at least part of their partition-theoretic sum (or $z_{1} \cup z_{2}$ ) (Smith, 1991). On the other hand, note that if we analogously define the partition-theoretic product $(\mathrm{z}=$ $\mathrm{z}_{1} \cap \mathrm{z}_{2}$ ) of two units within a given partition as the largest subunit shared in common by $\mathrm{z}_{1}$ and $\mathrm{z}_{2}$ - that is, as their greatest lower bound with respect to the subunit relation - then it turns out that this coincides with the mereological product already defined above. Mereological sum and product apply to both units and objects, while partition-theoretic sum applies to units only. The following symbols are used for the two groups of relations:

Figure 2: Symbols for Relations of Partition-theory and Mereology

\begin{tabular}{|l|c|c|}
\hline Sum & $\begin{array}{c}\text { Partition-theoretic } \\
\text { (for units) }\end{array}$ & $\begin{array}{c}\text { Mereological } \\
\text { (for units and for objects) }\end{array}$ \\
\hline Product & $\cup$ & + \\
\hline Inclusion & $\subseteq$ & $*$ \\
\hline Proper Inclusion & $\subset$ & $\leq$ \\
\hline
\end{tabular}


When restricted to units within a given partition, $\subseteq$ and $\leq$ coincide, and so do $\cap$ and $*$. We can think of $\subseteq$ as the result of restricting $\leq$ to the natural units picked out by the partition in question. We can think of set theory as amounting to the overlooking of the idea that there is a distinction between natural units and arbitrary unions. Set theory, indeed, derives all its power from this overlooking.

\subsection{Trees}

Philosophers since Aristotle have recognized that the results of our sorting and classifying activities can be represented as those sorts of branching structures which mathematicians nowadays call trees. For an example of a tree, see Figure 3, which represents the top level of the Foundational Model of Anatomy (FMA) ontology (see FMA, also Rosse and Mejino, 2007).

Trees are directed graphs without cycles (i.e., if we move along its edges, then we will always move down the tree and in such a way that we will never return to the point from which we started). They consist of nodes or vertices, and of directed edges, that connect the nodes. That the edges are directed means that the vertices connected by an edge are related to each other in a way that is analogous to an ordered pair; the relation between nodes is asymmetric or unidirectional. Here, we are interested specifically in rooted trees, that is, trees with a single topmost node to which all other vertices are connected, either directly or indirectly, via edges. In a rooted tree, every pair of vertices is connected by one and only one chain (or sequence of edges). We shall think of the directedness of an edge as proceeding down the tree from top to bottom (from ancestors to descendants). The connection between partitions and trees will now be obvious, as it is a simple matter to show that every finite partition can be represented as a rooted tree of finite depths, and vice versa (Mark, 1978). 
Figure 3: Highest-level Branches of the Tree Representing the FMA

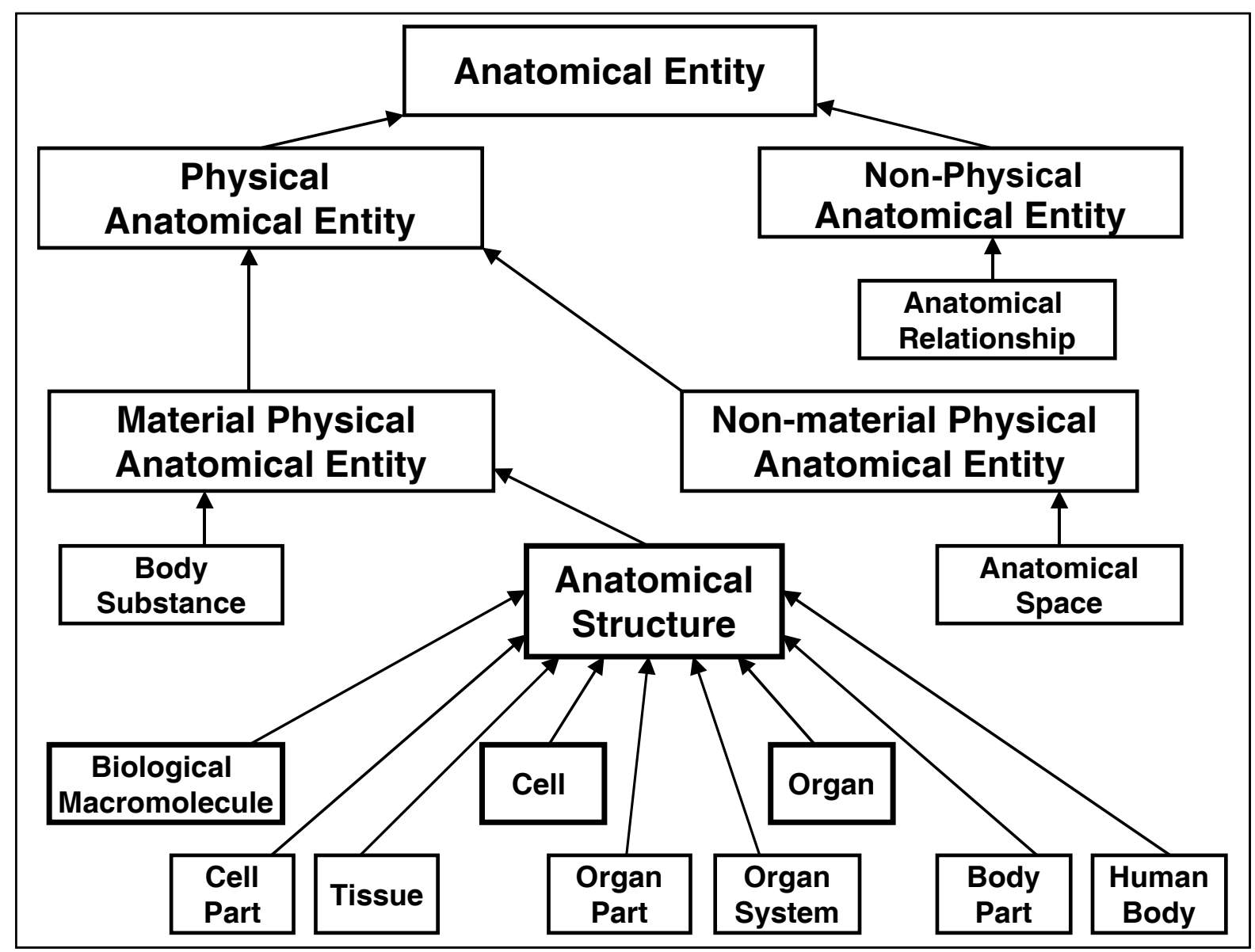

\section{Granular Partitions in their Projective Relation to Reality}

\subsection{Projection}

Partitions are more than just systems of units. They are constructed to serve as inventories or pictures or maps of specific portions of reality and, in this way, they are analogous to pictures or windows (Smith, 2001a). They are also analogous to propositions (Elementarsätze) as described by Wittgenstein in the Tractatus (1961). A proposition, for Wittgenstein, is built out of simple signs (names) arranged in a certain order. Each name, Wittgenstein tells us, stands in a projective relation to a corresponding object in the world; it cannot fail to strike its target. If a proposition is true, then its simple signs stand to each other within the proposition as the corresponding objects stand to each other in the world. It is in this sense that a true atomic proposition is a picture of a state of affairs in reality, as Wittgenstein puts it. That a proposition is a complex of names arranged in 
a certain order is, in our present context, equivalent to the thesis that a partition is a complex of units arranged in a certain order.

A partition is a complex of units in its projective relation to the world (compare Tractatus, 3.12). This relation may be effected either directly by the user of the partition - for example, in looking through the units of the grid and recording what objects are detected on the other side - or indirectly, with the help of proper names or other referring devices such as systems of coordinates or taxonomic labels.

From the perspective of granular partition theory projection may fail, and a partition may be such that there are no objects for its units to project onto (like the partition cataloguing Aztec gods). Here, however, we are interested primarily in partitions which do not project out into thin air in this way. In what follows, we shall assume that a unique projection is defined for each partition. In a more general theory, we can weaken this assumption by allowing projections to vary with time while the partition remains fixed, for example (this is allowed in Smith and Brogaard, 2002). Such variation of projection for a fixed partition is involved in all temporally extended sampling activity. Consider, for example, what happens when we use a territorial grid of units to map the presence of one or more birds of given species in given areas from one moment to the next.

\subsection{Location}

If projection is successful, then we shall say that the object upon which a unit is projected is located in that unit. The use of the term 'location' reflects the fact that one important inspiration of our work is the study of location relations in spatial contexts. One motivating example of a location relation of the sort here at issue is the relation between a spatial object such as a factory building and the corresponding rectangular icon on a map. Other motivating examples are of a non-spatial sort and include the relation between an instance (Tibbles) and its kind (cat).

In what follows, we make the simplifying assumption that objects are located precisely at their units. That is, we will assume that the boundaries of the real-world objects correspond to the boundaries of the partitions through which we apprehend them. Compare the way in which your brother Norse is exactly located at the unit 'Norse' in your partition (list) of your family members. In a more general theory, we liberalize the location relation in such a way as to allow for partial or rough location as well (Casati and Varzi, 1995; Bittner and Stell, 1998); as, for example, between 
a factory building and the corresponding square formed by the grid on a map.

\subsection{Transparency}

When projection succeeds, location is what results. Projection and location thus correspond to the two directions of fit - from mind to world and from world to mind - between an assertion and the corresponding truthmaking portion of reality. (For seminal work in this area, see Searle, 1983; compare also Smith, 1999.) Projection is like the relation which holds between your shopping list and the items which, if your shopping trip is successful, you will actually buy. Location is like the relation which obtains between the items you have bought and the new list your mother makes after your return, as she checks off those items which you have in fact succeeded in bringing back with you.

The formula ' $\mathrm{L}(\mathrm{o}, \mathrm{z})$ ' abbreviates: object $\mathrm{o}$ is located at unit $\mathrm{z}$. (And again: where this is required we can write ' $\mathrm{L}_{\mathrm{A}}(\mathrm{o}, \mathrm{z})$ ' for: $\mathrm{o}$ is located at $\mathrm{z}$ in partition A.) Location presupposes projection; an object is never located in a unit unless that object has already been picked out as the target of the projection relation associated with the relevant partition. But successful projection - by which is meant the obtaining of the projection relation between a unit and an object - also presupposes location, so that where both location and projection obtain they are simply the converse relations of each other.

We have now reached the point where we can formulate the first of our master conditions on partitions from the perspective of theory (B):

MB1: If object o is located at unit $\mathrm{z}$, then unit $\mathrm{z}$ projects onto object $\mathrm{o}$.

MB2: If unit $\mathrm{z}$ projects onto object $\mathrm{o}$, then object $\mathrm{o}$ is located at unit $\mathrm{z}$.

(Successful) projection and (successful) location are simple converses of each other. MB1 and MB2 tell us that a partition projects a given unit onto a given object if and only if that object is located in the corresponding unit. Very many partitions - from automobile component catalogues to our maps of states and nations - have this quality without further ado.

We shall call partitions which satisfy MB1 and MB2 transparent partitions, a notion which we can define as follows: 
DTr: Partition A is transparent if and only if, for every unit $\mathrm{z}$ of partition A and for every object o:

(i) if $\mathrm{z}$ projects onto $\mathrm{o}$, then $\mathrm{o}$ is located at $\mathrm{z}$, and

(ii) if $\mathrm{o}$ is located at $\mathrm{z}$, then $\mathrm{z}$ projects onto $\mathrm{o}$.

MB1 and MB2 jointly ensure that objects are actually located at the units that project onto them. Notice that, according to our definition, a transparent partition may still have empty units. Such units may be needed to leave room for what may be discovered in the future or to cover up for temporary lapses in memory.

\subsection{Functionality Constraints (Constraints Pertaining to Correspondence to Objects)}

\subsubsection{Projection is Functional: The Confused Schoolboy}

The property of transparency is still rather weak. Thus, transparency is consistent with ambiguity on the side of the units in relation to the objects they target. Such is the case where one unit projects onto two distinct objects. An example of the sort of problem we have in mind is the partition created by a lazy schoolboy studying the history of the Civil War in England. This partition has just one unit labeled 'Cromwell', and so it does not distinguish between Oliver and his son Richard.

Although such ambiguous units do sometimes exist, in an ideal scenario they should be rectified when they are discovered. To eliminate such ambiguity, we lay down a requirement that each partition must be such that its associated projection is a functional relation:

MB3: If unit $\mathrm{z}$ projects onto object $\mathrm{o}_{1}$ and onto object $\mathrm{o}_{2}$, then $\mathrm{o}_{1}$ and $\mathrm{o}_{2}$ are identical.

Figure 4: Transparent Partitions in which Projection is not Functional (left) and Location is not Functional (right)
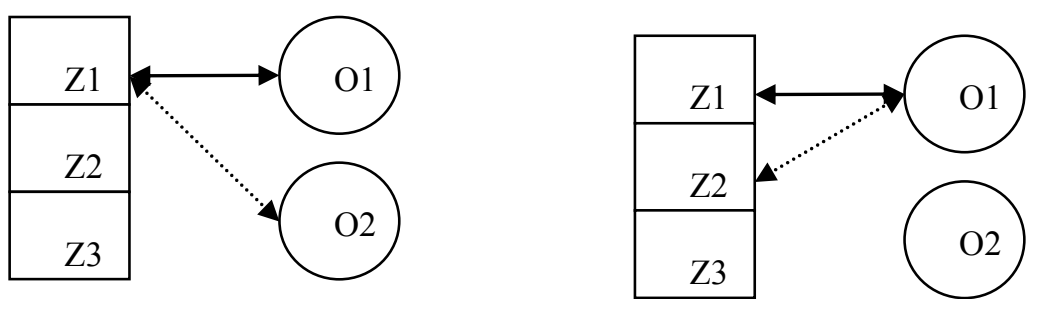
For partitions satisfying MB3, units are projected onto single objects. Consider the left part of Figure 4. The dotted arrow can occur in partitions satisfying merely MB1 and MB2 but not in partitions also satisfying MB3. Notice, though, that projection might still be a partial function, since MB3 does not rule out the case where there are empty units.

\subsubsection{Location is Functional: The Morning Star and the Evening Star}

Consider a partition with a maximal unit labeled 'heavenly bodies' and three subunits labeled 'The Morning Star', 'The Evening Star', and 'Venus', respectively. As we know, all three subunits project onto the same object. This partition is perfectly consistent with the conditions we have laid out thus far. Its distinct subunits truly, though accidentally, project onto the same object. However, a good partition should clearly be one in which such errors of duplication of representational units are avoided.

Partitions manifesting the desired degree of correspondence to objects in this respect must in other words be ones in which location, too, is a functional relation:

MB4: If the same object is located in unit $z_{1}$ and in unit $z_{2}$, then units $z_{1} z_{2}$ are identical.

MB4 ensures that location is a function, i.e., that objects are located at single units (one rather than two). Consider the right part of Figure 4. The dotted arrow can occur in partitions satisfying MB1 and MB2, not however in partitions also satisfying MB4. As MB3 rules out co-location, so MB4 rules out co-projection. Note that natural analogues of co-location and coprojection cannot be formalized within a set-theoretic framework.

\section{Correspondence of Mereological Structure}

Even in the presence of MB3 and MB4, MB1 and MB2 tell us only that if a unit in a partition projects upon some object, then that object is indeed located in the corresponding unit. They do not tell us what happens in case a unit fails to project onto anything at all. Thus, MB1-4 represent only a first step along the way towards an account of correspondence to reality for partitions. Such correspondence will involve the two further dimensions of structural mapping and of completeness. 


\subsection{Recognizing Mereological Structure}

An object o is recognized by a partition if and only if the latter has a unit in which that object is located (Smith and Brogaard, 2000). Intuitively, recognition is the partition-theoretic analogue of the standard setmembership relation. To impose a partition on a given domain of reality is to foreground certain objects and features in that domain and trace over others. Partitions are granular in virtue precisely of the fact that a partition can recognize an object without recognizing all its parts.

Partitions are designed to reflect the part-whole structure of reality through the fact that the units in a partition are themselves such as to stand in relations of part to whole. Given the master conditions expressed within the framework of theory (A) above, partitions have at least the potential to reflect the mereological structure of the domain onto which they are projected. In felicitous cases this potential is realized.

That we distinguish between the recognition (foregrounding, selection) of objects, on the one hand, and the reflection of mereological structure, on the other hand, is not an arbitrary matter. In Tractarian semantics, we distinguish between projection and isomorphism. The former obtains where we have some representation (for example a list or a map) which is intended to capture the entities in some domain of reality, while the latter obtains where this intention is fulfilled, so that there is a one-one correspondence between the units of the representation and the entities in the represented domain. In set theory we distinguish, for any given set, between a domain of elements and the set-theoretic structure imposed on this domain. Just as it is possible to have sets consisting entirely of Urelemente (together with a minimal amount of set-theoretic packaging), so it is possible to have partitions built exclusively out of minimal units (and one maximal unit). Such partitions amount, simply, to lists of the things that are recognized by their units, with no mereological structure on the side of these objects being brought into account.

Figure 5 (a) and 5 (b) represent partitions consisting of two minimal units $z_{1}$ and $z_{2}$ projecting onto objects $o_{1}$ and $o_{2}$. Case (a), a simple list, is unproblematic. Case (b) we shall also allow as unproblematic. This is in keeping with the notion that minimal units are the (relative) atoms of our system, and we take this to mean that they should be neutral with regard to any mereological structure on the side of their objects. An example of type (b) would be a list of regions represented at a conference to discuss measures against terrorism, a conference including representatives from 
both Germany and Bavaria. Here we are not concerned about the fact that Bavaria is a part of Germany.

Figure 5: Transparent Partitions with More or Less Desirable Properties
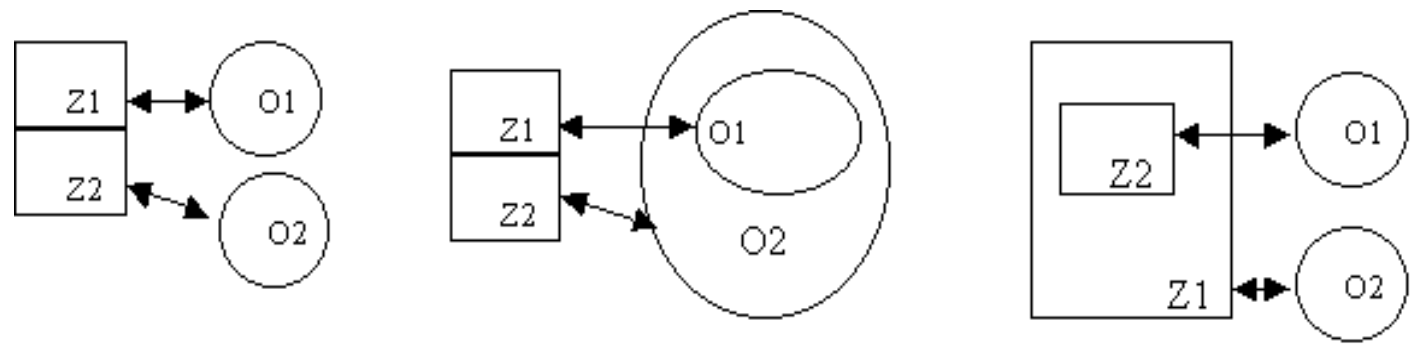

Cases like (c), in contrast, represent projections in which, intuitively, something has gone wrong. All three cases satisfy the master conditions we have laid down thus far, for the latter allow both for disjoint units to be projected onto what is not disjoint (b) and also for disjoint objects to be located in units which are not disjoint (c). Cases like (c) seem to fly in the face of a fundamental principle underlying the practice of hierarchical classification, namely, that objects recognized by species lower down in a hierarchical tree should be included as parts in whatever is recognized by the genera further up the tree. To exclude cases like (c), we shall impose a condition that mereological structure within a partition should not misrepresent the mereological relationships between the objects onto which the corresponding units are projected. We first of all define the following relation of representation of mereological structure between pairs of units:

DS1: Units $z_{1}$ and $z_{2}$ represent the mereological structure of the objects onto which they project if and only if, for objects $\mathrm{o}_{1}$, and $\mathrm{o}_{2}$ : if $\mathrm{o}_{1}$ is located in $\mathrm{z}_{1}$ and $\mathrm{o}_{2}$ is located in $\mathrm{z}_{2}$, and if $\mathrm{z}_{1}$ is a subunit of $\mathrm{z}_{2}$, then $\mathrm{o}_{1}$ is part of $\mathrm{o}_{2}$.

If $z_{1}$ is a subunit of $z_{2}$ then any object recognized by $z_{1}$ must be a part of any object recognized by $z_{2}$.

DS2: A partition is mereologically structure-preserving if and only if: each pair of units within the partition satisfies DS1.

We can now impose a new master condition:

MB5: All partitions are mereologically structure-preserving in the sense of DS2. 
Note that even MB5 is still very weak. Its effect is entirely negative, since it merely ensures that partitions do not misrepresent the mereological relationships between their objects. Partitions might still be entirely blind to (i.e. they may trace over) such relationships. Two minimal units might project onto objects which stand to each other in any one of the possible mereological relations (identity, proper parthood, disjointedness, overlap), and all pairs of units are likewise neutral as to the mereological relations between the objects onto which they are projected, provided only that they do not stand to each other in the subunit relation. This means that, given such units, we are entitled to infer nothing at all about the mereological relations among the corresponding objects.

Consider, for example, a partition that contains two units that recognize, respectively, mammals and whales. Suppose that this is a partition constructed at a time when the status of whales as mammals was not yet recognized. The unit labeled whales is not, then, included as a subunit of the unit labeled mammals. But the partition can still satisfy our conditions laid down so far. This is so, for example, if the unit that recognizes whales is a subunit of the unit recognizing animals but not a subunit of any other subunit of the unit recognizing animals (Partition $A_{1}$ in Figure 6). If the unit that recognizes whales were also a subunit of the unit that recognizes fish, for example, then the partition would misrepresent the mereological relationship between these two species and so violate MB5 (Partition $\mathrm{A}_{2}$ in Figure 6).

Figure 6: Partition $A_{1}$ does not misrepresent the mereological structure of the underlying domain. Partition $A_{2}$ places whales incorrectly in relation to fish and mammals.

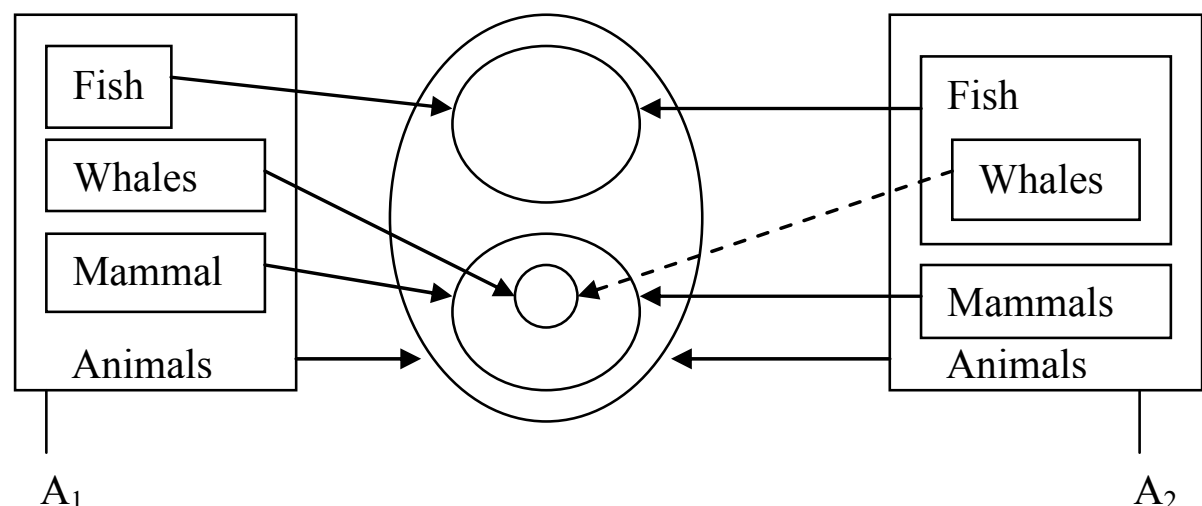

Partitions may trace over mereological relationships between the objects they recognize, but MB5 is strong enough to ensure that, if a partition tells us something about the mereological relationships on the side of the 
objects which it recognizes, then what it tells us is true. Notice that partition $\mathrm{A}_{2}$ still satisfies MB1-4.

Consider a domain of objects consisting of two regions, $\mathrm{x}$ and $\mathrm{y}$, that overlap in the region $\mathrm{v}$, so that the product of $\mathrm{x}$ and $\mathrm{y}$ is $\mathrm{v}$, with $\mathrm{v}$ being a proper part of $\mathrm{x}$ and of $\mathrm{y}$. (In symbols: $\mathrm{x} * \mathrm{y}=\mathrm{v}$ with $\mathrm{v}<\mathrm{x}$ and $\mathrm{v}<\mathrm{y}$.) Consider now a partition with units $\mathrm{z}_{1}$ and $\mathrm{z}_{2}$ recognizing $\mathrm{x}$ and $\mathrm{y}$, respectively, so that $\mathrm{x}$ is located in the first and $\mathrm{y}$ in the second unit: $\mathrm{L}(\mathrm{x}$, $\left.z_{1}\right)$ and $L\left(y, z_{2}\right)$. Assume further that $z_{1}$ and $z_{2}$ do not stand in any subunit relations to each other. Only four possibilities regarding the representation of $\mathrm{v}$ now remain: (1) our partition does not recognize $\mathrm{v}$ at all; (2) it recognizes $\mathrm{v}$ but traces over its mereological relationships to $\mathrm{x}$ and $\mathrm{y}$; (3) it recognizes $\mathrm{v}$ through a subunit of $\mathrm{z}_{1}$ but it traces over the fact that $\mathrm{v}$ could equally well be recognized by a subunit of $z_{2}$; (4) it recognizes $v$ through a subunit of $z_{2}$, but it traces over the fact that $v$ could equally well be recognized by a subunit of $z_{1}$. The fifth possibility - of allowing sub-units of both $z_{1}$ and $z_{2}$ to recognize $v-$ is excluded by the tree structure of granular partitions.

Let $\mathrm{x}$ and $\mathrm{y}$ be two neighboring countries which disagree about the exact location of their common boundary and let $\mathrm{v}$ be the disputed area. The inhabitants of country $\mathrm{x}$ consider $\mathrm{v}$ to be part of $\mathrm{x}$, the inhabitants of country y consider $v$ to be part of y. Possibility (1) then corresponds to the view of some third country at the other side of the globe which recognizes the countries $\mathrm{x}$ and $\mathrm{y}$ but does not care about their border dispute. (2) corresponds to the view of an observer who recognizes that there is a disputed area but who is neutral about the status of the disputed area. (3) corresponds to the view of country $x$ and (4) to that of country $y$.

Another example of case (2) is provided by Germany and Luxemburg, which overlap at their common border on the River Our. The river is part of both countries. Mapmakers normally have no facility to represent cases such as this, and so they either adopt the policy of not representing such common regions at all (the border is represented as a line which we cannot properly in this case imagine as being without thickness), or they recognize the region constituted by the river on the map but trace over its mereological properties. Larger-scale maps often embrace a third alternative, which is to misrepresent the relations between Germany and Luxenburg by drawing the boundary between the two countries as running down the center of the river. 


\subsection{The Domain of a Partition}

That upon which a partition is projected is a certain domain of objects in reality (the term 'domain' being understood in the mereological sense, i.e. as a mereological sum of entities in some region of reality demarcated in some way, for example on the basis of our focus of interest). We shall conceive the domain of a partition as the mereological sum of the pertinent objects, those upon which the partition sets to work: thus it is stuff conceived as it is prior to any of the divisions or demarcations effected by the partition itself. The domains of partitions will comprehend not only individual objects and their constituents, but also groups or populations of individuals (for example biological species and genera) as well as their constituent parts or members. Domains can comprehend also extended regions (bounded continua) of various types. Spatial partitions, for example maps of land use or soil type (Frank et al., 1997), are an important family of partitions with domains of this sort.

We are now able to specify what we mean by 'domain of a partition'. Our representation of partitions as trees and our condition on reflection of structure (MB5) ensure that all partitions trivially reflect the fact that the objects recognized by their units are parts of some mereological sum. For MB5 is already strong enough to ensure that everything that is located at some unit of a partition is part of what is located at the corresponding maximal unit.

We can thus define the domain of a partition simply as the object (mereological whole) onto which its maximal unit is projected. By functionality of projection and location there can be only one such object.

DD: The domain of partition $\mathrm{A}$ is identical to the object upon which its maximal unit is projected.

We now demand as a further master condition that every partition have a non-empty domain in the sense of DD:

MB6: For every partition A, there is an object $\mathrm{x}$ which is identical to the domain of partition A.

We then say that a partition represents its domain correctly if and only if MA1-5 and MB1-6 hold. Correct representations, as we see, can be highly partial. 


\subsection{The Granularity of Granular Partitions}

A correct representation is not necessarily a complete representation. Indeed, since partitions are cognitive devices, and cognition is not omniscient, it follows that no partition is such as to recognize all objects. This feature of partitions is captured by the following condition:

FP: There is no partition which projects onto everything in the sense that its maximal unit projects onto the universe as a whole and it has a subunit recognizing every object there is.

This condition does justice to the fact that the complexity of the universe is much greater than the complexity of any single cognitive artifact. This feature of partiality is captured already by our terminology of granular partitions. Partitions characteristically do not recognize the proper parts of the whole objects which they recognize.

It is the units of a partition which carry this feature of granularity with them. Like singletons in set theory, they recognize only single whole units, the counterparts of set-theoretic elements or members. If a partition recognizes not only wholes but also one or more parts of such wholes, then this is because there are additional units in the partition which do this recognizing job. Consider, for example, a partition that recognizes human beings and has units that project onto John, Mary, and so forth. This partition does not recognize parts of human beings - such as John's arm or Mary's shoulder - unless we add extra units for this purpose. Even if a partition recognizes both wholes and also some of their parts, it is not necessarily the case that it also reflects the mereological relationships between the two.

Partitions are cognitive devices which have the built-in capability to recognize objects and to reflect certain features of those objects' mereological structure and to ignore, or trace over, other features of this structure. We can now see that they can perform this task of tracing over in two ways, namely, (1) by tracing over mereological relations between the objects which they recognize, and (2) by tracing over, or failing to recognize, parts of those objects. Unless there is some smallest unit, (2) is a variety of tracing over that must be manifested by every partition. A third type of tracing over becomes apparent when we remember that partitions are partial in their focus, and thereby each partition traces over everything that lies outside its domain (here, we leave to one side the Spinoza 
partition, namely, the monad, which consists of just one all-encompassing universal unit).

Consider a simple biological partition of the animal kingdom including just one single unit, projecting on the species dog (canis familiaris). Our definition of the domain of a partition and our constraint on functionality of projection imply that, besides the species dog, also your dog Fido, and also Fido's DNA-molecules, are parts of the domain of this partition. But the latter are of course not recognized by the partition itself.

Partition theory hereby allows us to define a new, restricted notion of parthood that takes granularity into account. This restricted parthood relation is an analogue of partition-theoretic inclusion, but on the side of objects:

DRP: $\quad \mathrm{x}$ is a part of $\mathrm{y}$ relative to partition $\mathrm{A}$ if and only if $\mathrm{x}$ is recognized by a subunit of a unit in A which recognizes $\mathrm{y}$.

From DRP we can infer by MB5 that $\mathrm{x}$ is a part of $\mathrm{y}$ also in the unrestricted or absolute sense.

The usual common-sense (i.e., non-scientific) partition of the animal kingdom contains units recognizing dogs and mammals, but no units recognizing DNA molecules. Relative to this common-sense partition, DNA molecules are not parts of the animal kingdom in the sense defined by DRP, though they are of course parts of the animal kingdom in the usual, mereological sense of 'part'.

\section{Structural Properties of Correct Representations}

What are some of the more fundamental varieties of those partitions which satisfy the master conditions set forth above? We can classify such partitions according to: (1) the degree to which they match the structure of the objects which they represent (i.e., their structural fit); (2) their degree of completeness and exhaustiveness with respect to their domain; (3) their degree of redundancy (the smaller the redundancy the more adequate the representation). (For more on this, see Ceusters and Smith, 2006; and Smith, Kusnierczyk, Schober, and Ceusters, 2006). 


\subsection{Structural Fit}

We require of partitions that they at least not misrepresent the mereological structure of the domain they recognize. This leaves room for the possibility that a partition is merely neutral about (traces over) some or all aspects of the mereological structure of its target domain. Taking this into account, we can order partitions according to the degree to which they actually do represent the mereological structure on the side of the objects onto which they are projected. At the maximum degree of structural fit, we have those partitions which completely reflect the mereological relations holding between the objects which they recognize.

Such a partition satisfies a condition which is the weak converse of MB5:

CM: If object $\mathrm{o}_{1}$ is part of object $\mathrm{o}_{2}$, and if both $\mathrm{o}_{1}$ and $\mathrm{o}_{2}$ are recognized by a partition $A$, then the unit at which $o_{1}$ is located is a subunit of the unit at which $\mathrm{O}_{2}$ is located.

A partition satisfying CM is mereologically monotonic: it recognizes all the restricted parthood relations obtaining in the pertinent domain of objects. A very simple example is given by a flat list projected one-for-one upon a collection of disjoint objects.

\subsection{Completeness}

So far we have allowed partitions to contain empty units, i.e., units that do not project onto any object. We now consider partitions which satisfy the constraint that every unit recognizes some object:

$\mathrm{CC}$ : If $\mathrm{z}$ is a subunit of partition $\mathrm{A}$, then there is some object $\mathrm{o}$ which is located at $\mathrm{z}$.

We say that partitions that satisfy CC project completely. Of particular interest, however, are partitions that project completely and in such a way that projection is a total function (partitions which satisfy both MB3 and CC). An example is a map of the United States representing its constituent states (with a whole for the District of Columbia). There are no non-states within the territory projected by such a map and every unit projects uniquely onto just one state. 


\subsection{Exhaustiveness}

So far we have accepted that there may be objects in our target domain which are not located at any unit. This feature of partitions is sometimes not acceptable: governments want all their employed citizens to be located in some unit of their partition of taxable individuals. They want their partitions to satisfy a completeness constraint to the effect that every object in the domain is indeed recognized. In this case we say that location is complete, or that the partition exhausts its domain. We might be tempted to apply the following axiom to capture the exhaustiveness constraint:

(*) If object o is included in the domain onto which partition A projects, then there is a unit $\mathrm{z}$ at which $\mathrm{o}$ is located.

However, this condition is unrealizable because not every object is recognized in every partition: the tax authorities do not (as of this writing) want to tax the separate molecules of their citizens, and so the partitions of reality which they employ do not recognize these molecules.

It will in fact be necessary to formulate several restricted forms of exhaustiveness, each one of which will approximate in different ways the condition expressed in $(*)$.

One such exhaustiveness condition might utilize a sortal predicate (schema) $\varphi$ that singles out the kinds of objects our partition is supposed to recognize (for example, in the case of the just-mentioned partition, the predicate being a taxable individual). We now demand that the partition recognize all of those objects in its domain which satisfy $\varphi$ :

$\mathrm{CE}_{\varphi}$ If object $\mathrm{o}$ is included in the domain onto which partition A projects, and if o satisfies requirement $\varphi$, then there is a unit $\mathrm{z}$ at which o is located.

We can very simply use any predicate to define a partition over any domain, by the following definition:

Object $\mathrm{o}$ is located in unit $\mathrm{z}$ of partition $\mathrm{A}$ if and only if:

(i) $\mathrm{o}$ is properly included in the domain onto which A projects and

(ii) o satisfies requirement $\varphi$.

Hence $\mathrm{CE}_{\varphi}$ entails the completeness of one partition relative to another.

The intuition behind constraints like $\mathrm{CE}_{\varphi}$ is to catch everything above a certain resolution at least once. If you want your partition to rule out 
locating an item in two separate units, so that no item is counted twice, then you want the partition to satisfy $\mathrm{CE}_{\varphi}$ and MB4. You may also want to make sure that your partition does not include empty units, which you rule out by imposing condition $\mathrm{CC}$. For partitions that satisfy the criteria $\mathrm{CE}_{\phi}$, and $\mathrm{MB} 1-5$, and $\mathrm{CC}$ projection and location are total functions (relative to the selected predicate $\varphi$ ) and one is the inverse of the other. Notice that neither of the following holds:

$$
\begin{aligned}
& (* *) \text { if } \mathrm{MB} 4 \text { and } \mathrm{CE}_{\phi} \text { and } \mathrm{CC} \text {, then MB3 } \\
& (* * *) \text { if MB3 and } \mathrm{CE}_{\phi} \text { and } \mathrm{CC} \text {, then MB4 }
\end{aligned}
$$

Counterexamples are given in Figure 7 (a) and (b), respectively, where each depicted object is assumed to satisfy $\varphi$.

Figure 7: Functionality of Projection and Location are Independent of Completeness and Exhaustiveness.

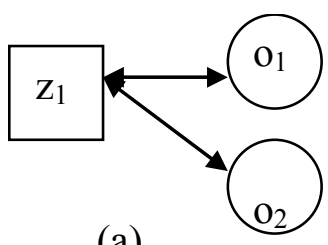

(a)

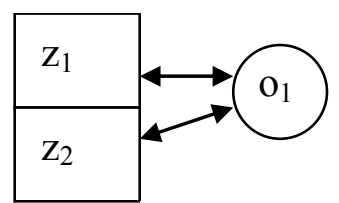

(b)

\subsection{Comprehension Axioms}

The following is the partition-theoretic equivalent of the unrestricted settheoretic comprehension axiom. For each predicate $\varphi$ there is a partition $\mathrm{A}(\varphi)$ whose location relation is defined as follows:

There is a unit in partition A in which object o is located if and only if o satisfies $\varphi$.

Under what conditions on $\varphi$ can this be allowed? One type of restriction that is relevant to our purposes would allow $\varphi$ to be unrestricted but would affirm additional restrictions on objects, for example in terms of spatial location. Thus we might define a family of spatial partitions $\mathrm{A}(\varphi, \mathrm{r})$, where $r$ is some pre-designated spatial region, in such a way that:

There is a unit in partition A in which object o is located if and only if:

(i) o satisfies $\varphi$, and

(ii) $\mathrm{o}$ is spatially located in region $\mathrm{r}$. 
Something like this is in fact at work in the partitions used by epidemiologists, ornithologists, and others who are interested in (types of) objects at specific sites.

\subsection{Redundancy}

Partitions are natural cognitive devices used by human beings to serve various practical purposes. This means that partitions normally will be called upon to avoid certain sorts of redundancy. Here, we distinguish what we shall call correspondence redundancy and structural redundancy. Correspondence redundancy occurs where we have too many disjoint units in a partition. Structural redundancy occurs where two or more non-disjoint units are present which project onto the same portion of reality. Necessarily, empty units (such as would be defined by the predicate 'entity that is not identical with itself') represent one type of correspondence redundancy which is excluded by condition CC. Another type of correspondence redundancy is present in a partition with two distinct units whose labels would tell us ex ante that they must necessarily project upon the very same object. Clearly, and most simply, a partition should not contain two distinct units with identical labels.

The following case is not quite so trivial. Consider a partition with a unit labeled vertebrates which occurs as a subunit of the unit labeled chordates in our standard biological classification of the animal kingdom. Almost all chordates are in fact vertebrates. Suppose (for the sake of the illustration) that biologists were to discover that all chordates must be vertebrates. Then such a discovery would imply that, in order to avoid structural redundancy, they would need to collapse into one unit the two units (of chordates and vertebrates) which at present occupy distinct levels within their zoological partitions. A constraint designed to rule out such structural redundancy would be:

CR: A unit in a partition never has exactly one immediate descendant.

This rules out partition-theoretic analogues of the set theorist's $\{\{a\}\}$.

\section{Fullness and Cumulativeness}

Thus far we have distinguished completeness and exhaustiveness. We now introduce a third type of comprehensiveness factor for partitions, which is needed for ensuring that the successive levels of the partition relate to each 
other in the most desirable way. We can initially divide this third type of completeness into two sub-types, namely, fullness and cumulativeness. Fullness requires that each unit $\mathrm{z}$ have enough subunits (which are immediate descendants) to fill out $\mathrm{z}$ itself. Cumulativeness requires that these immediate subunits be such that the objects onto which they are projected are sufficient to exhaust the domain onto which the containing unit is projected. Fullness, accordingly, pertains to theory (A), cumulativeness to theory (B).

Non-fullness and non-cumulativeness represent two kinds of shortfall in the knowledge embodied in a partition. Non-fullness is the shortfall which arises when a unit has insufficiently many subunits within a given partition (for instance it has a unit labeled mammal, but no subunits corresponding to many of the species of this genus). Non-cumulativeness is the shortfall which arises when our projection relation locates insufficiently many objects in the units of our partition, for example when I strive to make a list of the people that I met at the party yesterday, but leave out all the Welshmen. Fullness and cumulativeness are satisfied primarily by artificial partitions of the sorts constructed in database environments. In the remainder of this section we assume, for the sake of simplicity, that there are no redundancies in the sense of CR.

\subsection{Fullness}

Consider a partition with three units labeled: mammals, horses, and sheep. This partition is transparent, by our definition (DTr); but falls short of a certain sort of ideal completeness. We can express the problem as follows.

In set theory, if a collection of subsets of some given set forms a partition of this set in the standard mathematical sense, then these subsets are (1) mutually exhaustive and (2) pairwise disjoint (the latter meaning that the subsets have no elements in common). An analogue of condition (2) holds for minimal units in our present framework, since minimal units are always mereologically disjoint (they cannot, by definition, have subunits in common). Condition (1) however does not necessarily hold within the framework of partition theory. This is because, even where the partition-theoretic sum of minimal units is identical to the maximal unit, the minimal units still do not necessarily exhaust the partition as a whole. The mereological sum $(+)$ of units is, we will recall, in general smaller than their partition-theoretic sum $(\cup)$. 
Thus, we can define a unit as full within a given partition if its subunits are such that their mereological sum and their partition-theoretic sum coincide.

DFull: Unit $\mathrm{z}$ is full if and only if: the mereological sum of its subunits is identical to the partition-theoretic sum of its subunits.

However, DFull does not suffice to capture the intended notion of fullness for partitions. To see the problem, consider the partition consisting of

\begin{tabular}{|c|c|c|c|}
\hline \multicolumn{3}{|c|}{ cells } \\
\hline \multicolumn{2}{|c|}{ prokaryotic } & \multicolumn{2}{c|}{ eukaryotic } \\
\hline nucleoid & $\begin{array}{c}\text { ribosomes as only } \\
\text { organelles }\end{array}$ & nucleus & $\begin{array}{c}\text { membrane bound } \\
\text { organelles }\end{array}$ \\
\hline
\end{tabular}

The unit in the top row satisfies DFull, but it is not full relative to all of its subunits, since the mereological sum of the units nucleoid and ribosomes as only organelle is not identical to the unit prokaryotic. The problem arises because, if $\mathrm{x}$ is mereologically included in $\mathrm{y}$, then the mereological sum of $\mathrm{x}$ and $\mathrm{y}$ is $\mathrm{y}$; and if $\mathrm{x}$ is partition-theoretically included in $\mathrm{y}$, then the partition-theoretic sum of $\mathrm{x}$ and $\mathrm{y}$ is $\mathrm{y}$. From this, it follows that only the immediate subunits of a given unit $z_{1}$ contribute to its mereological and partition-theoretic sums.

This, however, tells us what we need to take into account in defining what it is for a unit to be full relative to all its subunits within a given partition A, namely that each of its constituent units must be full relative to its immediate descendents. This yields:

DFull*: Unit $\mathrm{z}_{1}$ is a full* unit of partition $\mathrm{A}$ if and only if, for every $\mathrm{z}$ : if $\mathrm{z}$ is a partition-theoretic part of $z_{1}$, then $z$ is either a full unit of $A$ or a minimal unit of $A$.

Here minimal units have been handled separately because they do not have subunits. One can see that, while cells in the mentioned partition are full, they are not full*, because the units prokaryotic and eukaryotic are neither full nor minimal.

Thus far we have defined fullness for units. We can now define what it means for a partition to be full, as follows:

DFull2: A partition is full if and only if all its non-minimal units are full (or, equivalently, all its units are full*). 
Notice that full partitions might in principle contain empty units, which may or may not have subunits.

\subsection{Cumulativeness}

Cumulativeness plays the same role in theory (B) which fullness plays in theory (A). The intuitive idea is as follows: a unit is cumulative relative to its immediate subunits if the mereological sum of the objects onto which those subunits project (for short: the units' projection) is identical to the projection of their partition-theoretic sum. For non-empty and non-minimal units with at least two immediate subunits we define:

DCu1: A unit $\mathrm{z}$ is cumululative if and only if: the partition-theoretic sum of its projection is identical to the mereological sum of its projection.

One can see that, under the given conditions, it is the case that the projection of a unit's partition-theoretic sum is identical to the projection of the unit. Consequently:

If a unit $\mathrm{z}$ is cumulative, then the mereological sum if its projections is identical to $z$, and vice-versa.

Again, the cumulative condition ensures that $z_{1}$ is cumulative relative to its immediate subunits. In order to ensure cumulativeness of a unit with respect to all its subunits, we define:

DCu2: A partition is cumulative if and only if all its units are cumulative.

$\mathrm{DCu}^{*}$ : A partition is cumulative* if and only if all its units are either cumulative or minimal.

\subsection{Equivalence of Fullness and Cumulativeness}

From the definitions above, it follows that cumulative partitions for which CC and MB1-5 hold are full and that they do not contain empty units. To see why this is so, consider a non-minimal unit $\mathrm{z}_{1}$ of which it holds that its partition-theoretic sum is identical to its projection.

We need to show that in this case $z_{1}$ itself is identical to its partitiontheoretic sum. Since by MB5 projection does not distort mereological structure, two cases need to be considered: 
(a) $\mathrm{z}_{1}$ and its partition-theoretic sum are mereologically disjoint, which means that they trace over the mereological relationships between $z_{1}$ 's projection and the projection of its partition-theoretic sum; and

(b) $\mathrm{z}_{1}$ 's partition-theoretic sum is mereologically included in $\mathrm{z}_{1}$, and $\mathrm{z}_{1}$ is mereologically included in $z_{1}$ 's partition-theoretic sum, in which case the mereological relationships between $z_{1}$ 's projection and the projection of its partition-theoretic sum are preserved.

In case (b), $\mathrm{z}_{1}$ is identical with its partition-theoretic sum, as desired. Case (a) cannot occur, however, since projection is a one-one mapping; that is, distinct units project onto distinct objects (by CC and MB1-4) and, therefore, the two distinct and disjoint units $\mathrm{z}_{1}$ and its partition-theoretic sum cannot project onto one and the same object, as is required by our assumption that the projection of $z_{1}$ is identical to the partition-theoretic sum of $z_{1}$ 's projection.

We can also show that, in the opposite direction, full partitions for which CC and MB1-5 hold are cumulative. To see this, assume that $z_{1}$ is a non-minimal unit in a full partition $A$. We then need to show that if $z_{1}$ is identical to its partition-theoretic sum, then $z_{1}$ 's projection is identical to the projection of its partition-theoretic sum. Assume that $z_{1}$ is identical to its partition-theoretic sum. We need to consider two cases:

(a) $\mathrm{Z}_{1}$ 's partition-theoretic sum is a unit in partition A;

(b) $\mathrm{z}_{1}$ 's partition-theoretic sum is not a unit in partition A.

In case (a), the following holds: By MB5 we have: $z_{1}$ 's projection and the projection of its partition-theoretic sum are mereologically included in each other. Since the underlying unit-structure is full, the projection of $z_{1}$ 's partition-theoretic sum is identical to the partition-theoretic sum of $z_{1}$ 's projection. Thus, $z_{1}$ 's projection and the partition-theoretic sum of its projection are mereologically included in one another. Hence they are, as desired, identical.

In case (b), $z_{1}$ 's partition-theoretic sum is not a unit in the partition A. Consequently it is as if there is some extra entity $\mathrm{x}$ (which we will characterize in the next section as what we shall call 'empty space') which, together with $z_{1}$ 's partition-theoretic sum, sums up to $z_{1}$. Consequently $z_{1}$ and its partition-theoretic sum cannot be identical. This, however, contradicts our assumption of fullness. Therefore case (b) cannot occur. 
It follows that the notions of fullness and cumulativeness are logically equivalent for completely projecting partitions. We can accordingly distinguish just two classes of such partitions:

(1) Full and cumulative

Sample partition: a list of the 50 US states, divided into two sub-lists: the contiguous 48, the non-contiguous 2.

Objects: the states themselves.

Projection: the obvious 'Utah'-Utah projection relation.

(2) Non-full and non-cumulative

Sample partition: you have a terrible hangover, and your accounting of the people at the party consists of four units: John, Mary, the Irish, the Welsh. As it happens, Sally is Scottish.

A cumulative partition $\mathrm{A}$ is also exhaustive in the sense of $\left(\mathrm{CE}_{\varphi}\right)$, with $\varphi$ requiring that there be a unit which is a part of $\mathrm{A}$ and which projects onto an object. Full partitions are also mereologically monotonic (CM). To see this, assume that it holds that $\mathrm{z}_{1}$ 's projection is mereologically included in $\mathrm{z}_{2}$ 's projection. Two cases need to be considered:

(a) $z_{1}$ and $z_{2}$ are mereologically disjoint and trace over the relationships between their respective projections; or

(b) $z_{1}$ and $z_{2}$ are not mereologically disjoint in which case, by MB5, the mereological relations are preserved.

Consider case (a). If $z_{1}$ and $z_{2}$ are not subunits of each other we have their mereological sum not being equivalent to $z_{2}$. Therefore, by fullness, we have $z_{1}$ 's and $z_{2}$ 's partition-theoretic sums, when mereologically added, not equaling $z_{2}$ 's partition-theoretic sum. Since fullness implies cumulativeness, we have the partition-theoretic sum of $z_{1}$ 's projection, when mereologically added to the partition-theoretic sum of $z_{2}$ 's projection, not being equal to the partition-theoretic sum of $z_{2}$ 's projection; and the mereological sum of $z_{1}$ 's projection and $z_{2}$ 's projection not being equal to $z_{2}$ 's projection. Hence we arrive at a contradiction of our previous assumption: $\mathrm{z}_{1}$ 's projection is not mereologically included in $\mathrm{z}_{2}$ 's projection. Therefore case (a) cannot occur. In case (b) preservation of mereological structure immediately yields $z_{1}$ being partition-theoretically included in $\mathrm{z}_{2}$, which is the desired result. 


\subsection{Empty Space}

When a unit falls short of fullness, it successfully projects onto some given domain, but its subunits do not succeed in projecting onto the entirety of this domain. It is then as if there is some extra but invisible component in the unit, in addition to its subunits. We shall call this additional component 'empty space' (noting that the term 'empty' here has a quite different meaning from what it has in the phrase 'empty unit' as used above). Consider the partition depicted in Figure 8:

Figure 8: A Partition with Empty Space.

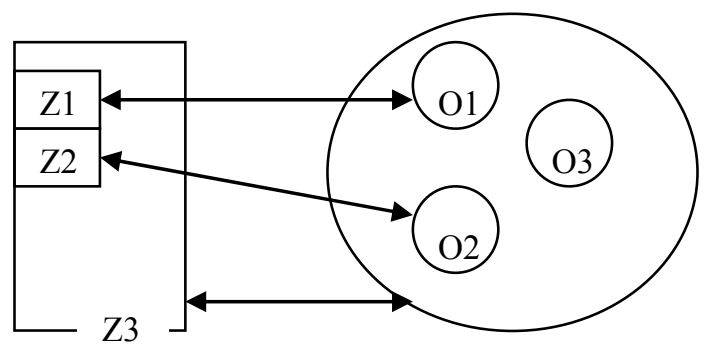

Here, the empty space is that part of $z_{3}$ which is not occupied by $z_{1}$ and $z_{2}$. Notice that this empty space is a component of $z_{3}$ but it is neither itself a unit nor is it made up of units. Empty space is that part of a unit which is not covered by its subunits; it is a zone within a unit where no subunits are to be found, reflecting, for example, the fact that many taxonomies are incomplete at lower levels.

Another characteristic of empty space is that there must be something that potentially fills it. In our case this means: more subunits. Empty space is inert in the sense that it does not project onto anything. Empty space is normally hidden to the user of the partition in which it exists, for otherwise this user would surely have constructed a fuller partition. In some cases however a user might deliberately accept empty space in order to have the means of acknowledging that something has been left out. Alternatively, the existence of empty space in a given partition might be brought to the attention of the user. We point in a certain direction and ask: What is there? The theory of empty space hereby serves as the starting-point for an ontology of questions (Schuhmann and Smith, 1987); empty space corresponds to a hole in our knowledge. 


\subsection{Fullness and Emptiness}

Recall that, if $z_{1}$ is a full unit, then it is identical to its partition-theoretic sum, and vice versa. This means that, if $z_{1}$ is not a full unit, then its partition-theoretic sum is properly included in it; and that, if this is the case, then it is not a full unit. Consequently we can define what it means for $\mathrm{x}$ to be the empty space of the unit $\mathrm{z}_{1}$ as follows. We first of all define $\mathrm{x}$ fills $\mathrm{z}_{1}$ :

DFills: Empty space $x$ fills unit $z_{1}$ if and only if:

(i) $z_{1}$ is not a full unit, and

(ii) $\mathrm{z}_{1}$ is identical the mereological sum of $\mathrm{x}$ and $\mathrm{z}_{1}$ 's partition-theoretic sum.

The empty space in $z_{1}$ is then $z_{1}$ 's smallest filler, and we define:

DES: $x$ is the empty space in $z_{1}$ if and only if

(i) $x$ fills the space not occupied by the subunits of $z_{1}$ and

(ii) $\mathrm{x}$ is disjoint from all subunits of $\mathrm{z}_{1}$.

We note, in passing, that minimal units, on the basis of the definitions above, are either empty or they are completely made up of empty space. ('Minimal' means: there is no further knowledge available, within a given partition, as concerns the objects onto which minimal units are projected.) Intuitively it is clear that DES determines the empty space of a unit uniquely. Consider $\mathrm{x}$ and $\mathrm{y}$, which are both empty spaces in unit $\mathrm{z}$ as defined by DES. Each then fills the space not occupied by z's subunits, and are disjoint from all of z's subunits. Thus $\mathrm{x}$ and $\mathrm{y}$ are identical.

\subsection{Empty Space and Knowledge}

The presence or absence of empty space is a dimension of a granular partition that is skew to the dimension pertaining to the existence of empty units. An empty unit is a unit that fails to project. Empty space is that in a partition which leaves room for the addition of new knowledge.

Figure 8 depicts a partition of the animal kingdom consisting of three units, where $z_{3}$ recognizes the animal kingdom as a whole, $z_{1}$ recognizes dogs, and $z_{2}$ recognizes cats. $z_{3}$ is the partition-theoretic sum of $z_{1}$ and $z_{2}$, but the sum of the projections of $z_{1}$ and $z_{2}$ is less than $z_{3}$. New units can be 
inserted into the partition if new species are discovered (e.g., the species indicated by $\mathrm{O}_{3}$ ).

There is at least one other sort of knowledge shortfall which we shall need to consider in a complete theory of partitions. This arises when there are missing levels within a partition-theoretic hierarchy. A partition of the phylum arthropoda which mentions all of its species and genera but leaves out the units for its classes (e.g. insecta) is an example of this sort of incompleteness.

\section{Identity of Granular Partitions}

As a step towards a definition of identity for partitions, Smith and Brogaard (2003) propose a partial ordering relation between partitions, which they define as follows:

Partition A is mereologically included in partition B if and only if every unit of $\mathrm{z}$ is also a unit of $\mathrm{B}$.

They then define an equivalence relation on partitions as follows:

Partition A is identical to partition B if and only if:

(i) A is mereologically included in B, and

(ii) $\mathrm{B}$ is mereologically included in $\mathrm{A}$.

Now, however, we can see that a definition along these lines will work only for partitions which are full. What, then, of those partitions which are equivalent in the sense of DE but not full? What are the relationships between the presence of empty and redundant units and the question of the identity of partitions? And what is the bearing on the question of identity of the phenomenon of empty space? Can partitions that have empty or redundant units be identical? Can partitions which are not full be identical?

The question of whether or not partitions that have empty or redundant units are identical cannot be answered without a theory of labeling. If corresponding empty units in two distinct partitions are to be considered as identical, they need to have at least the same labels. We can only address this question informally here.

Consider the partition of the people in your building according to number of days spent behind bars. You can construct this partition prior to undertaking any actual inquiries as to who, among the people in your building, might be located in its various units. Thus, even before carrying 
out such inquiries, you can know that this is a more refined partition than, for example, the partition of the same group of people according to number of years spent behind bars. The two partitions are distinct, and they will remain distinct even if it should turn out that none of the people in your building has spent any time at all in jail. In both cases, all the people in your building would then be located in a unit labelled zero, and all the other units in both partitions would be empty. Yet the two partitions would be nonetheless distinct, not least because their respective maximal units would have different labels.

We can now return, briefly, to our question whether partitions that are neither full nor cumulative can be said to be identical. One approach to providing an answer to this question would be to point out that, even though two partitions are outwardly identical, they might still be such that there are different ways to fill the corresponding empty space. Suppose we have what are outwardly the same biological taxonomies used by scientists in America and in Australia at some given time, both with the same arrays of empty units. Suppose these partitions are used in different ways on the two continents, so that, in the course of time, their respective empty space gets filled in different ways. Were they still the same taxonomy at the start?

\section{Conclusions}

This essay is a first step towards a formal theory of granular partitions. We defined master conditions that need to be satisfied by every partition. These master conditions fall into two groups, namely, (A) master conditions characterizing partitions as systems of units, and (B) master conditions describing partitions in their projective relation to reality.

At the level of theory (A) partitions are systems of units that are partially ordered by the subunit relation. Such systems of units can always be represented as trees; they have a unique maximal component and they do not have cycles in their graph-theoretic representations. But partitions are more than just systems of units. They are also cognitive devices that are directed towards reality.

Theory (B) takes this latter feature into account by characterizing partitions in terms of the relations of projection and location. Units in partitions are projected onto objects in reality. Objects are located at units when projection succeeds. To talk of granular partitions is to draw attention to the fact that partitions are in every case selective; even when they recognize some objects, they will always trace over others. 
Partitions are also capable of reflecting the mereological structure of the objects they recognize through a corresponding mereological structure on the side of their array of units. This does not mean, however, that all partitions actually do reflect the mereological structure of the objects they recognize. For an important feature of partitions is that they are also capable of tracing over (ignoring) mereological structure.

Our discussion of granularity showed that partitions have three ways of tracing over mereological structure: (1) by tracing over mereological relations between the objects which they recognize; (2) by tracing over the parts of such objects; and (3) by tracing over the wholes which such objects form. The tracing over of parts is (unless mereological atomism is true) a feature manifested by every partition, for partitions are in every case coarse grained. The tracing over of wholes reflects the property of granular partitions of foregrounding selected objects of interest within the domain onto which they are projected and of leaving all other objects in the background where they fall in the domain of unconcern.

And the relevance to this book? Nearly everything that has been said about ontologies and their relation to reality in this volume can be illuminated by conceiving ontologies as granular partitions standing in a relation of projection to the entities their terms denote. 